\title{
Associative Memory Inspired by Antibody Dynamics
}

\author{
[ Chung-Ming $\mathrm{Ou}$ ]
}

\begin{abstract}
Immune memory of antigens can be defined as a limit behavior of immune networks with antibody dynamics. Immune memory mechanism is studied by combining network structure and chaotic dynamical systems. The latter is contributed to defining affinity index of antibody chain. Associative memory can be explored by antibody dynamics determined by such affinity indices.
\end{abstract}

Keywords - idiotypic immune network, antibody dynamics, antibody chain, associative memory, Lyapunov exponent

\section{Introduction}

System perspectives of immune responses have been studied by Nowak and May [15]. Immune memory on the basis of immune network theory was proposed by Jerne [12]. The memory of each previously invaded antigen is distributed through the immune network. Moreover, immune systems can be regared as complex adaptive systems [12]. On the other hand, immune memory mechanism have been extensively analyzed in terms of sparse and distributed associative memory[2][5][18].

Many researches have been proceeded according to the immune network theory. Perelson has proposed the shape space analysis for the immune network theory [17]. Carneiro et al. have discussed immune networks based on cooperations of B-and T-cells [7]. The process of antibody concentration variations can describe dynamics of idiotypic immune network. It is an ongoing research topic to exploit relationship between immune memory and internal image which is regarded as a part of format of antigenic memory [6][10]. Some closed loop network structure, namely cyclic idiotypic immune network (CIIN), can explain the existence of such antigen even its clones are completely vanished.

In this research, we build a mathematical model of immune memory mechanism, which describes the memory cells with network dynamics. It is achieved by simplifying idiotypic immune networks to some antibody chains. One advantage of antibody chains is its simplicity to define the network dynamics. We explore the immune memory mechanism based on antibody dynamics. There are two issues: (1) simplified architecture of idiotypic immune network proposed by [19]; (2) associative memory based on statistical immunodynamics inspired by [1][3][14].

One question for antibody dynamics is as follows. What is the dynamics invoked by those antibodies ? One direction for this issue is the dynamics of cross-reactive immune response. Morita has proposed non-monotonic dynamics for associative memory [14] which introduced the autocorrelation matrix. Our research is inspired by such statistical dynamics for antibody chain, particularly, discrete chaotic dynamical system defined by logistic function. The memory format of the corresponding antigen can be defined as limiting behavior of atibody dynamics.

\section{Research Background}

\section{A. Immune Memory}

Immune systems react rapidly to the same or similar (mutated) antigens which had invaded the same human body before. They can" memorize" associatively the formations of previously invaded antigens. Some variables related to the immune memory might be correlated to the antibody concentration according to computational biology.

Immune memory mechanism is not fully understood so far. Recent viewpoint of memory cells is that they are not lived longer than virgin cells; their life cycles depend on the persistence of antigens [13]. On the other hand, researches based on immune network theory imply the immune memory mechanism is formed by cyclic idiotypic immune networks (CIINs) than specific memory cells [19]. It is important to explain how memory recalls are activated for similar antigenic invasions. Therefore, it is worth of considering the immune network theory for such associative memory mechanism. In particular, its role in immune memory can be analyzed by network dynamics. We can mathematically describe the formation of an immune memory, and the recall process of such memory. The antibody chain has been proposed as a central aspect of the associative properties of immune memory [9][18].

\section{B. Antibody Chain}

One major character of idiotypic network theory is that the immune systems will emulate the presence of antigens even after they are eliminated [18]. IINs can be represented by antibody $A b_{i}$ (Figure 1). The advantage of this simplified network, namely, antibody chain, is the following. The immune memory, can be exploited by state transitions determined by antibody chains. There are two stable states for idiotypic immune network; for the first state, the antibody $A b_{1}$ is not produced. For the second stable state, $A b_{1}$ is produced and antigen may or may not be completely eliminated [16]. Network models of immune responses described by ordinary differential equations can be referred to [8][20][21]

Antibody dynamics can be inspired by some immune network models such as the one proposed in [19]. Such antigen-antibody interactions can be a long sequence, namely, an antibody chain, which is defined as follows. For an idiotypic immune network $\left.\left\{\left\{J_{i}\right\}_{i}\right\}_{-1}^{N}, M\right\rangle$, an antibody chain $A C=\left\{A b_{1}, A b_{2}, \cdots A b_{N}\right\}$ is defined as follows. (1) $A b_{i} \in$ $L U_{i}$, for all $i=1,2, \cdots N$; (2) The idiotype of $A b_{i}$ can be recognized by the partatope of $A b_{i+1}$, namely, $A b_{i} \rightarrow A b_{i+1}$, for all $i=1,2, \cdots N-1$; (3) $A b_{N} \rightarrow A b_{1}$. 


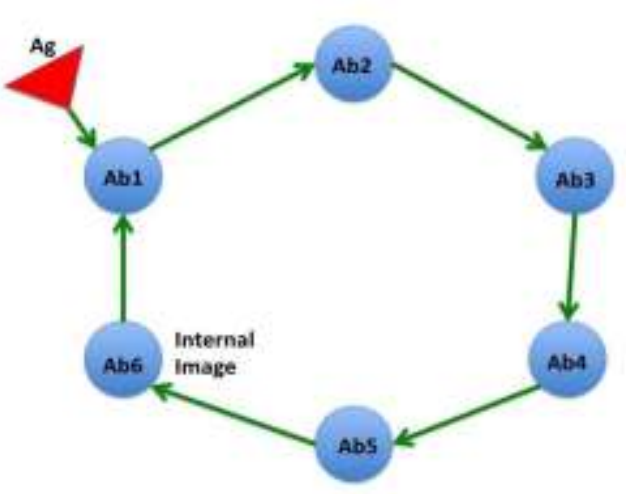

Figure 1. Antibody Chain

\section{One-dimensional Chaotic Dynamical System}

A well-known one-dimensional chaotic system is the iteration generated by the logistic function which is defined as follows.

$$
F(x ; \alpha)=\alpha \cdot x(1-x)
$$

where $n=1,2, \cdots, x_{0} \in[0,1]$ is called the initial condition of (1). $\alpha \in[0,4]$ is the bifurcation parameter of (1). Such parameter is the major character for dramastic change of system behavior. Equation (1) also generates a onedimensional discrete dynamical system defined as follows.

$$
x_{n+1}=F\left(x_{n}\right)=\alpha \cdot x_{n}\left(1-x_{n}\right)
$$

$n=0,1, \cdots, x_{0}$ is called an initial condition of (2). Figure 2 is the bifurcation diagram of (2). It shows that the values of fixed points for (2) for varied $\alpha \in[0,4]$. While $1<\alpha<3$, the dynamics has two fixed points, one is 0 , the other is $x-\frac{a-1}{a}$. As $\alpha \approx 3.5$, periodic orbit with periodicity 2 appears. As $\alpha$ increases, periodic orbits with higher periodicities appear. As $\alpha>3.65$, (2) shows chaotic behavior

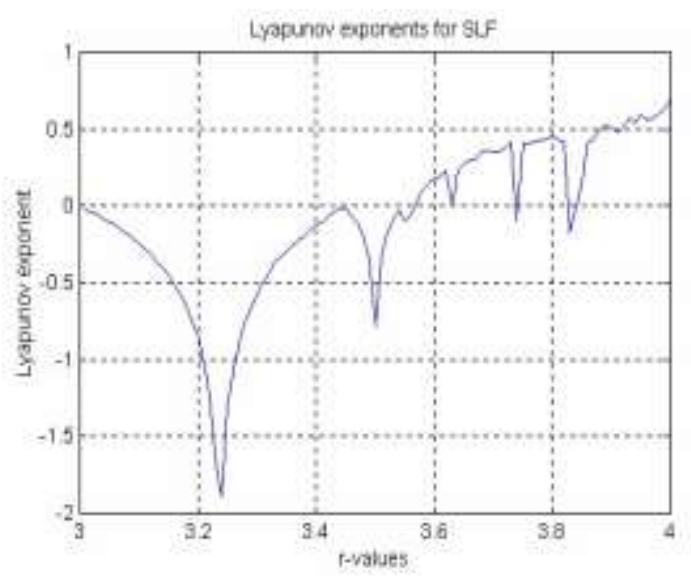

Figure 2. Lyapunov Exponents of (2)

The method of Lyapunov exponents, which is defined as follows, is one important way to determine whether an initial condition $x_{0}$ of (2) with parameter $\alpha$ will show chaotic behavior or not.

Definition 1. Let $\mathrm{f}: \mathrm{D} \subset \mathrm{R}^{1} \rightarrow \mathrm{R}^{1}$ be a real-valued continuous function. Consider the discrete dynamical system $x_{n+1}=f\left(x_{n}\right)$. The Lyapunov exponent of this system at $x_{0}$ is defined by

if the limit exists.

The Lyapunov exponents of (2) can be referred to Figure 2. We note that for $\alpha<3.65$, the Lyapunov exponent for any $x_{0} \in(0,1)$ is negative. As for $\alpha>0$, the Lyapunov exponents is greater than zero in general. However, some values are negative.

\section{Results}

We propose a model of immune memory mechanism based on the one-dimensional chaotic system (2); it is biologically-reasonable computation to transform antigenic format to memory format. Every antibody chain can induce some network dynamics which generate memory formats of antigens. This immune memory mechanism can explain the associative property on the basis of bifurcation parameter of (2). This model is verified by the associative memory of immune systems and the affinity index defined in this section.

\section{A. Network Dynamics of Antibody Chains}

A network dynamics $F$ of antibody chains inspired by the logistic function (1) is proposed. It is a type of state transition function which can generate immune memory of any given antigen. Some characters of the antibody chain $A C$ can influence the forming of immune memory. If an $A C$ has strong affinity for each pair of its adjacent antibodies $\left(A b_{i}\right.$, $\left.A b_{i+1}\right)$, then it will incur strong associative memory for antigens similar to previously invaded ones. Therefore, we define the affinity index of an antibody chain by the sum of affinities between all adjacent antibodies.

Definition 2. Let $\mathrm{n}$ be the length of molecules $\mathrm{X}$ and $\mathrm{Y}$. The the affinity between $\mathrm{X}$ and $\mathrm{Y}$, denoted by $\gamma(\mathrm{X}, \mathrm{Y})$, is defined by $1-\frac{a, X, Y}{n}$, where $d$ is the Hamming distance.

Definition 3. The affinity index $\mathrm{L}_{\mathrm{AC}}$ induced by an antibody chain $\mathrm{AC}$ is defined by

$$
L_{A C}=4 \cdot\left(1-\frac{1}{N-1}\left(\sum_{i=1}^{N-1} \gamma\left(A b_{i+1}, A b_{i}\right)\right)\right.
$$

$L_{A C}$ is a real number in $[0,4]$ which represents the average of affinities among pairs of adjacent antibodies $\left(A b_{i+1}, A b_{i}\right)$. Given an antigen $A g^{T}$ (column vector), it can be transformed to a real number $x_{0}$ between 0 and 1 . For example, if $A g=$ "11001", then $x_{0}=2^{-1}+2^{-2}+2^{-5}=$ 0.78125 .

The basic idea of the immune memory mechanism is that the degree of the associativity is proportional to some inner structure of antibody chain. Affinity index can be regarded as such inner strength. The smaller the index, the more associative the immune memory mechanism exhibits. This index computes ratio of the same attributes between adjacent antibodies. Now the network dynamics $F$ derived by the affinity index, $L_{A C}$, is as follows.

$$
\begin{aligned}
& \qquad x_{n+1}=F\left(x_{n} ; L_{A C}\right)=L_{A C} \cdot x_{n}\left(1-x_{n}\right) \\
& n=0,1,2, \cdots .
\end{aligned}
$$

$$
\lambda\left(x_{0}\right)=\lim _{n \rightarrow \infty} \frac{1}{n} \sum_{i=0}^{-1} \ln \left|f^{\prime}\left(x_{i}\right)\right|
$$




\section{B. Memory formed by Antibody Chains}

We define the immune memory function based on the antibody dynamics as follows.

Definition 4 . The immune memory function $\mathrm{f}: \mathrm{R} \rightarrow \mathrm{R}$ is a real-valued continuous function defined on $\mathrm{R}$ with the following condition. There exists some nonempty set $\mathrm{E} \subset \mathrm{R}$ such that $\lim _{\mathrm{k}} \rightarrow \infty \mathrm{f}^{\mathrm{k}}(\mathrm{x})$ exists for all $\mathrm{x} \in \mathrm{E}$.

Definition 5. Let $\gamma(\cdot, \cdot)$ represent the affinity between two binary molecule formats. An immune network $A C=\left\{A b_{i}\right\}_{i-1}^{N}$ activated by an antigen $\mathrm{Ag}$ is equipped with associative memory mechanism, if for $\epsilon \in(0,1)$, there exists some $\delta \in(0,1)$ and $\mathrm{k}_{0}$ a positive integer, such that whenever a new antigen $\mathrm{Ag}^{\prime}$ with $\gamma\left(\mathrm{Ag}, \mathrm{Ag}^{\prime}\right)>\delta$ implies that $\gamma\left(\mathrm{f}^{\mathrm{k}}(\mathrm{Ag}), \mathrm{f}^{\mathrm{k}}(\mathrm{Ag})\right)>\epsilon$.

For antibody dynamics, the limit behavior of $f^{k}(A g)$ depends on the affinity index of $A C$. For higher affinity threshold, the higher the possibility that the memory format of $\mathrm{Ag}$ is simply a stable equilibrium point. We have the following result for the immune memory mechanism. Let $L^{-}$ $\subset[0,4]$ be the region of parameter with negative Lyaponov exponents.

Proposition 1. Let $\mathrm{F}$ be logistic function (1). Then the immune memory of any antigen exists for all $\alpha \in \mathrm{L}^{-}$.

\section{Simulations}

We simulate memory forming process via network dynamics (3)-(4). We also analyze the memory formats of mutated antigens $A g^{\prime}$ which are similar to the previously invaded and memorized antigens $\mathrm{Ag}$ with only a few different attributes. The following algorithm describes the process of memory forming by antibody dynamics.

\section{Algorithm 1 Cross-reactive immune memory}

Input:

Size of_Pool: size of antibody pool

$\mathrm{Ag}$ : antigen

$A g^{\prime}:$ mutated antigen of $A g$

$k$ : $k$-level immune memory

$m$ : number of mutated attributes

$\lambda$ : threshold of affinity matching

Output:

$d_{k}$ : Overlapping difference between memory formats of $A g$ and $A g^{\prime}$

1. Randomly generating a pool of antibodies with fixed length $n$.

2. (Antibody Chain Forming) Randomly generating an antibody chain of $A g$, say $A C$

3. Calculating affinity index of $A C, L_{A C}$

4. Forming memory format of $A g$ by calculating $F^{k}\left(A g, L_{A C}\right)$

5. Memory recall of mutated antigen $A g^{\prime}$ by calculating

$F^{k}\left(A g^{\prime}, L_{A C}\right)$

6. $\mathrm{d}_{\mathrm{i}} \leftarrow \mathrm{F}^{\mathrm{i}}\left(\mathrm{Ag}, \mathrm{L}_{\mathrm{AC}}\right)-\mathrm{F}^{\mathrm{i}}\left(\mathrm{Ag}^{\prime}, \mathrm{L}_{\mathrm{AC}}\right)$

We realize that even for simple dynamics (3)-(4), it is very difficult to classify completely memory formats of varied antigens. One better way is the following. Rather than seeking different format of immune memories, we focus on classify the different types of "difference" between antigens and their mutations.

1) Simulation One: $1<L_{A C}<2$.: Figure 3 shows the memory formats of (randomly generated) $A g$ and its mutated antigen $A g^{\prime}(m=5, \lambda=0.7)$. Two memory formats are identical and equal to $0.025 . L_{A C}=1.0211$

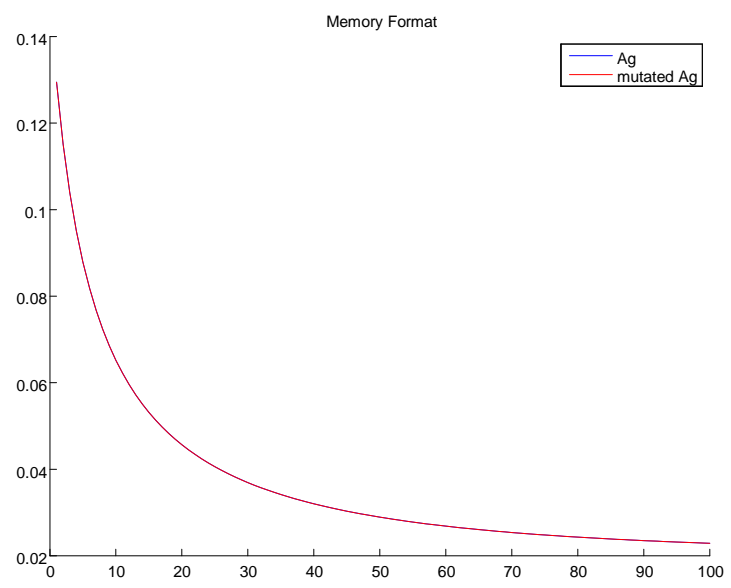

Figure 3. Memory Format for Some Antigen $\left(1<L_{A C}<2\right)$

2) Simulation Two: $2<L_{A C}<3$.: Figure 4 shows that memory format for $\mathrm{Ag}$ is equal to 0.55 . Its mutated antigen $A g$ induces a cross-reactive immune response activated by original antibody chain, as its memory format is also convergent to that of $A g . L_{A C}=2.20$

3) Simulation Three: $3<L_{A C}<3.6$. . We observe that $A C$ is difficult to form if affinity threshold $\lambda \geq 0.7$. Therefore we will simulate the memory format for $\lambda \geq 0.7$ by assigning $L_{A C}$ values directly to (4) without generating antibody chains.

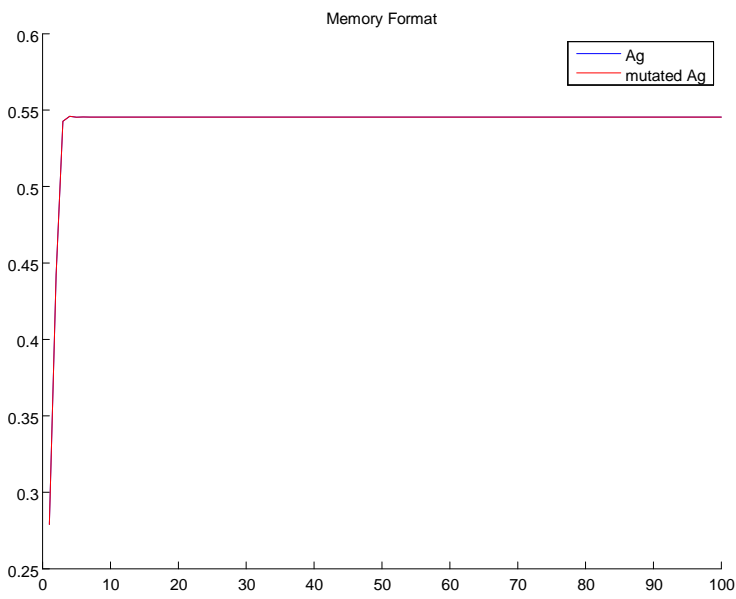

Figure 4. Memory Format for some Antigen $\left(2<L_{A C}<3\right)$

$\mathrm{L}_{\mathrm{AC}}=3.1026$. As for the memory format of mutated antigen $\mathrm{Ag}^{\prime}$, Figure 5 illustrates a better view that two memory formats are identical (after 30 iterations). The mutated antigen $\mathrm{Ag}^{\prime}$ induces a cross-reactive immune response activated by original antibody chain, as its memory format is also convergent to that of $\mathrm{Ag}$.

4) Simulation Four: $3.6 \leq L_{A C}<4$ : Now $L_{A C}=3.6$. As for the memory format of mutated antigen $A g^{\prime}$, Figure 6 
illustrates a better view that two memory formats are completely different. In this case, the corresponding $A C$ cannot activate a cross-reactive immune response to $A g^{\prime} ; A b_{1}$ cannot effectively eliminate $A g$ clones.

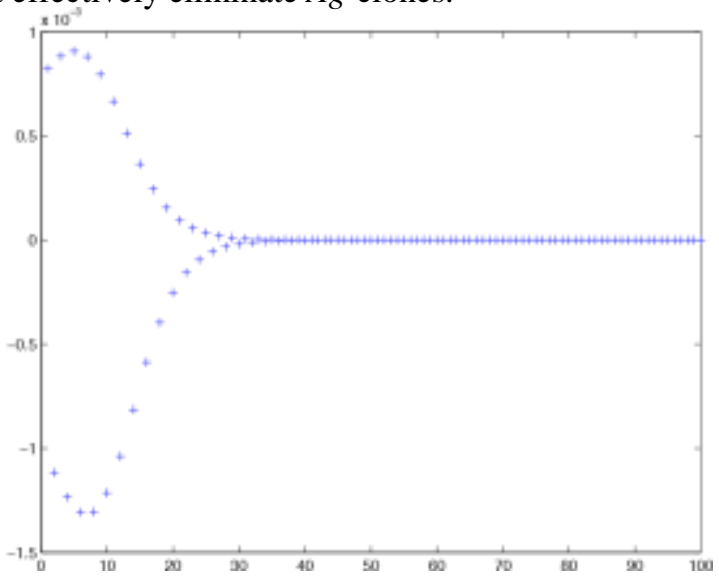

Figure 5. Difference of Memory Formats between $A g$ and $A g$

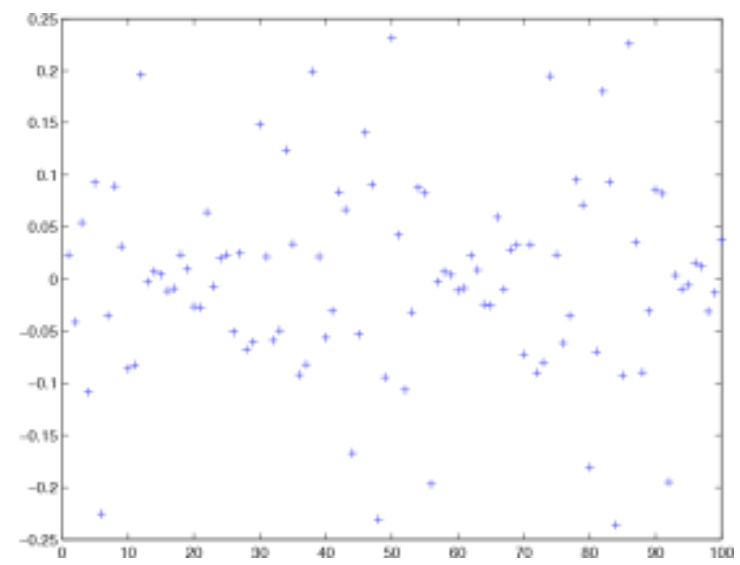

Figure 6. Difference of Memory Formats between $A g$ and $A g^{\prime}(L=3.6)$.

\section{Conclusions}

A model of antibody dynamics based on one dimensional chaotic dynamical system is proposed. It can describe the associativity of immune memory mechanism. The affinity index of antibody chain controls the bifurcation parameter of logistic function. Moreover, for higher affinity index, the immune network show associative memory for mutated antigens.

Our model focuses on the memory forming process which is unique while comparing to other researches. For example, Anderson et al. studied intensively about the immune network model with antibody dynamics based on Cayley tree [4]. For further research direction, we are interested in adopting such network structure of antibody dynamics to our model of memory forming. There are two aspects for such model; one is the memory format of an given antigen related to the limit behavior of the dynamical systems $F^{k}$ as $k \rightarrow \infty$. The other is the cross-reactive behavior of mutated antigen, namely $F^{k}\left(A g^{\prime}\right)$.

\section{Acknowledgment}

The author would like to thank support from the Ministry of Science and Technology of Taiwan under the grant number MOST-105-2221-E-424-002.

\section{References}

[1] F. Abbattista, G. D. Gioia, G. D. Santo, and A. Fanelli, "An asspciative memory based on the immune networks," in Proceedings of the IEEE Internatinal Conference on Neural Networks, 1996, 1996, pp. 519-523.

[2] E. Agliari, A. Barra, G. Ferraro, F. Guerra, and D. Tantari, "Anergy in self-directed b lymphocytes: A statistical mechanic perspective," J. Theoretical Biology, vol. 375, pp. 21-31, 2015.

[3] S.-I. Amari and K. Maginu, "Statistical neurodynamics of associative memory," Neural Networks, vol. 1, pp. 63-73, 1988.

[4] R. Anderson, A. Neumann, and A. Perelson, "A cayley tree immune network model with antibody dynamics," Bulletin of Mathematical Biology, vol. 55, pp. 1091-1131, 1993.

[5] A. Barra and E. Agliari, "Stochastic dynamics for idiotypic immune networks," Physica A, vol. 389, pp. 5903-5911, 2010.

[6] C. Bona, "Internal image concept revisited," Proceeding of the Society for Experimental Biology and Medicine, vol. 213, pp. 32-42, 1996.

[7] J. Carneiro, A. Coutinho, J. Faro, and J. Stewart, "A model of the immune networks with bt cell co-operation. i-prototypical structures and dynamics," J. Theoretical Biology, vol. 182(4), pp. 513-529, 1996.

[8] A. Coutinho, "A walk with francisco varela from first- to second generation networks: In search of the structure, dynamics and metadynamics," Biol. Res., vol. 36, pp. 17-26, 1993.

[9] J. Farmer, N. Packard, and A. Perelson, "The immune system, adaptation, and machine learning," Physica, vol. 22D, pp. 187-204, 1986.

[10] J. Fernandez, G. Acostra, and M. Mayosky, "From network-toantibody robustness in a bio-inspured immune system," BioSystems, vol. 104, pp. 109-117, 2011.

[11] G. Hoffman, "A theory of regulation and self-nonself discrimination in an immune network," Eor. J. Immunol., vol. 5, pp. 638-647, 1975.

[12] N. Jerne, "Towards a network theory of the immune system," Ann. Immunol. (Inst. Pasteur), vol. 125C, p. 373, 1974.

[13] P. Matzinger, "Memories are made of this ?" Nature, vol. 369 (23 June), pp. 605-606, 1994.

[14] M. Morita, "Associative memory with nonmonotone dynamics," Neural Networks, vol. 6, pp. 115-126, 1993.

[15] M. Novak and R. May, Virus Dynamics: Mathematical Principles of Immunology and Virology. Oxford University Press, 2001.

[16] G. Parisi, "An introduction to the immune network," arXiv, vol. http://arxiv.org/abs/cond-mat/9501046v1, 1995.

[17] A. S. Perelson, "Immune network theory," Immunological Reviews, vol. 10, pp. 5-36, 1989.

[18] D. Smith, S. Forrest, and A. Perelson, "Immunological memory is associative," Artificial Immune Systems and Their Applications,The international Conference on the Multi-Agent Systems Workshop Notes, Kyoto, 1996, pp. 62-70, 1999.

[19] T. Sonoda, "Formation and stability of a memory state in the immune network," J. Physical Society of Japan, vol. 81(4), pp. 1408-1424, 1992.

[20] P. Varela and A. Coutinho, "Second generation immune networks," Immunol. Today, vol. 12, pp. 159-166, 1991.

[21] G. Weisbuch, R. DeBoer, and A. Perelson, "Localized memories in idiotypic networks,” J. Theor. Biol., vol. 146, p. 483, 1990.

About Author (s):

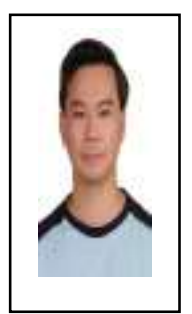

[Dr. Chung-Ming Ou is a full professor of Department of Information Management, Kainan University, Taiwan. His research interests include computational intelligence, artificial intelligence, machine learning and data science.] 